\title{
Development of $\alpha$-Amino Acid Detection with Peroxyoxalate Chemiluminescence by Using Water-Acetonitrile-Ethyl Acetate Mixed Solution
}

\author{
Hyo Kan1, Ryo Mizutani1, Kazuhiko Tsukagoshi'1,2* \\ ${ }^{1}$ Department of Chemical Engineering and Materials Science, Faculty of Science and Engineering, Doshisha University, \\ Kyotanabe, Japan \\ ${ }^{2}$ Bio-Microfluidic Science Research Center, Doshisha University, Kyotanabe, Japan \\ Email: ^ktsukago@mail.doshisha.ac.jp
}

How to cite this paper: Kan, H., Mizutani, R. and Tsukagoshi, K. (2020) Development of $\alpha$-Amino Acid Detection with Peroxyoxalate Chemiluminescence by Using Water-Acetonitrile-Ethyl Acetate Mixed Solution. American Journal of Analytical Chemistry, 11, 15-24.

https://doi.org/10.4236/ajac.2020.111002

Received: November 5, 2019

Accepted: January 18, 2020

Published: January 21, 2020

Copyright (อ 2020 by author(s) and Scientific Research Publishing Inc.

This work is licensed under the Creative Commons Attribution International License (CC BY 4.0).

http://creativecommons.org/licenses/by/4.0/

\begin{abstract}
Peroxyoxalate chemiluminescence was, for the first time, examined by using ternary mixed solutions of water-hydrophilic/hydrophobic organic solvent. Eosin $\mathrm{Y}$ as a model fluorescence compound was dissolved with the ternary solutions of water (1.0 mM carbonate buffer, $\mathrm{pH}$ 9.0)-acetonitrile-ethyl acetate, water-rich of 15:3:2 volume ratio and organic solvent-rich of 3:8:4 volume ratio, to which bis(2,4,6-trichlorophenyl) oxalate and hydrogen peroxide chemiluminescence reagent were added. The chemiluminescence observed with the ternary solutions, especially the organic solvent-rich solution, showed a larger signal than that observed with the water only solution or water-acetonitrile mixed solution. Chemiluminescence in the presence of twenty types of $\alpha$-amino acid was similarly examined by using the ternary organic solvent-rich solution. The chemiluminescence of three $\alpha$-amino acids with fluorescence properties was enhanced with the ternary solution. The data reported here may contribute to development of a new, sensitive peroxyoxalate chemiluminescence detection system.
\end{abstract}

\section{Keywords}

Peroxyoxalate chemiluminescence, $\alpha$-Amino Acids, Water-Acetonitrile-Ethyl Acetate, Bis(2,4,6-Trichlorophenyl) Oxalate

\section{Introduction}

Various types of aqueous-organic solvent mixed solutions are used in dissolu- 
tion [1], cleaning [2], preservation [3], and as reaction solvents [4]. Such mixed solutions are also useful in separation science, as extraction solvents and in liquid chromatography. We reported unique behavior of ternary mixed solutions of water-hydrophilic/hydrophobic organic solvent, such as water-acetonitrile-ethyl acetate mixed solution [5] [6] [7]. For example, the ternary mixed solutions were confirmed to work as a two-phase separation solution. The mixed solutions separated into upper and lower phases through the phase transformation in a batch vessel. In contrast, when the mixed solution was fed into a microspace, such as capillary tubes and microchannels on a microchip, annular flow with inner and outer phases was generated under certain conditions; we call this "Tube Radial Distribution Flow" (TRDF). The specific microfluidic flow, TRDF, was applied to capillary liquid chromatography [8] and micro-extraction [9].

The mixing behavior of ternary water-hydrophilic/hydrophobic organic solvent solutions was investigated using a Y-type microchannel. These two mixed solutions, water-acetonitrile and acetonitrile-ethyl acetate, were individually fed into two separate microchannels of a microchip that combined to form a single channel in a Y-type microchannel, affording a ternary mixed solution in the single channel. Unique microfluidic behavior was observed in the single channel. We termed it "microfluidic inverted flow" for the ternary mixed solutions [10]. Denaturation was examined in a ternary mixed solution of water-hydrophilic/ hydrophobic organic solvent using $\lambda$-DNA and a plasmid as models. The experimental data indicated that $\lambda$-DNA changed from a double helix structure to a single helix structure and that the plasmid partially transformed to generate a denaturation bubble in the structure. The novel idea of using the ternary mixed solution first enabled the interaction of the hydrophobic organic solvent molecule with the double helical structure of DNA [11].

In our previous study, we examined luminal chemiluminescence (CL) behavior in a ternary mixed solvent solution of water-acetonitrile-ethyl acetate, and the mixed solution was found to enhance luminol CL in time and intensity under certain conditions [12]. In this study, we examined CL behavior with four kinds of mixed solutions of water only, water-acetonitrile, and ternary mixed solutions of water-acetonitrile-ethyl acetate for peroxyoxalate reagent, instead of luminol reagent [13] [14], in the presence of Eosin Y as a model fluorescence compound. The peroxyoxalate CL of Eosin Y was found to be most enhanced by the ternary mixed solutions.

The CL detection methods of $\alpha$-amino acids without labeling procedures have been incorporated in flow injection analysis [15], high-performance liquid chromatography [16], and capillary electrophoresis [17]. They provided simple and procedures with high sensitivity. We tried to examine the peroxyoxalate $\mathrm{CL}$ response of $\alpha$-amino acids in the present batch-type CL measurement system with a ternary mixed solution in order to get basic data for developing a flow system in the future. 


\section{Experiment}

\subsection{Reagents and Materials}

Water was purified with an Elix UV 3 system (Millipore Co., Billerica, MA). All reagents used were commercially available and of analytical grade. Bis(2,4,6trichlorophenyl) oxalate (TCPO), Eosin $\mathrm{Y}$, hydrogen peroxide $\left(\mathrm{H}_{2} \mathrm{O}_{2}\right)$, acetonitrile, ethyl acetate, and twenty types of $\alpha$-amino acids were purchased from Wako Pure Chemical Industries, Ltd. (Osaka, Japan).

\subsection{Measurement System}

The CL profiles and CL counts were examined with a batch-type detection system (CLD-100FC; Tohoku Electronic Industrial Co. Ltd., Sendai, Japan). Here, the CL meant the total CL counts obtained during 480 seconds of measurement for CL. We used four kinds of solution as follows: (A) water only, (B) wateracetonitrile mixed solution (1:1, volume ratio), (C) the water-acetonitrile-ethyl acetate ternary mixed solution (15:3:2, volume ratio; water-rich), and (D) the water-acetonitrile-ethyl acetate mixed ternary solution (3:8:4, volume ratio; organic solvent-rich). In this study, the water was prepared as $1.0 \mathrm{mM}$ carbonate buffer, $\mathrm{pH}$ 9.0. The component ratios of the solutions are plotted in the phase diagram of the ternary mixed solution shown in Figure 1.

The preparation of CL regent solution and the mixing procedure of the reagent solution and the analyte solution containing fluorescent compounds, such as eosin Y, were carried out as a reference of our previous paper [18], as follows:

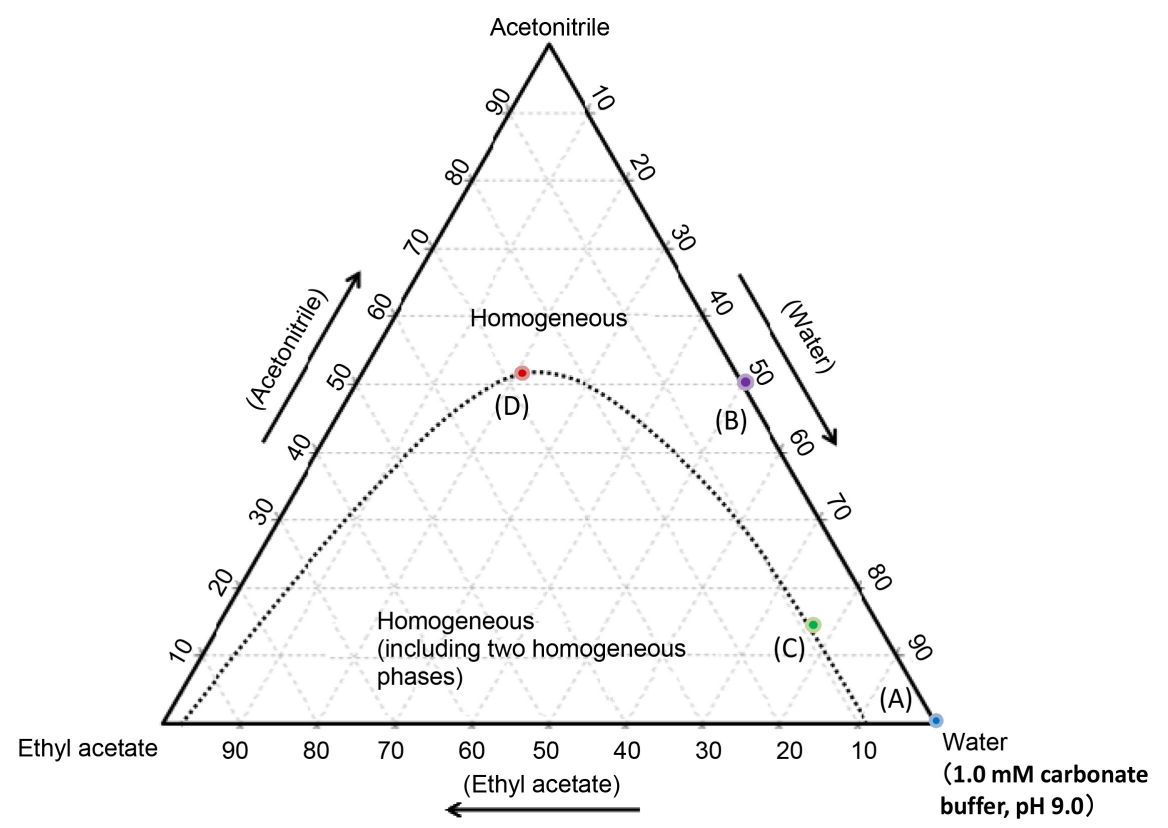

Figure 1. Phase diagram of the water-acetonitrile-ethyl acetateternary mixed solution at $22^{\circ} \mathrm{C}$. Solutions: (A) the water only, (B) the water-acetonitrile mixed solution (1:1, volume ratio), (C) the water-acetonitrile-ethyl acetate mixed solution (15:3:2, volume ratio; water-rich), (D) and the water-acetonitrile-ethyl acetate mixed solution (3:8:4, volume ratio; organic solvent-rich). 
the CL reagent solution (2.0 mM TCPO-200 $\mathrm{mM} \mathrm{H}_{2} \mathrm{O}_{2}, 0.05 \mathrm{~mL}$; acetonitrile was used as a solvent) was added to each of the above solutions $(2.5 \mathrm{~mL})$ containing Eosin $\mathrm{Y}$ in the petri dish, through a micro-syringe with constant stirring to obtain the CL profile. The CL was measured through a detection window of 3.1 $\mathrm{cm}^{2}$. Similarly, we examined CL profiles by using the solutions (A) and (D) containing $\alpha$-amino acids instead of Eosin Y, and measured the CL through a detection window of $15.9 \mathrm{~cm}^{2}$.

\section{Results and Discussion}

\subsection{CL of Eosin Y with the Four Solutions}

The phase diagram of water-acetonitrile-ethyl acetate is shown in Figure 1 together with the component ratios of the solutions (A)-(D). The dotted line indicates the boundary or solubility curve between homogeneous and heterogeneous solution. The CL profiles in the presence of Eosin Y were examined by using the solutions (A)-(D), by the CL measurement system. Figure 2 shows the CL profiles of $1.0 \mathrm{mM}$ Eosin $\mathrm{Y}$ with the solutions (A)-(D). As can be seen in the figure, the CL increased in the following order: (A), (B), (C), and (D). We also examined the CL counts of various concentrations of Eosin $\mathrm{Y}$ with the solutions (A)-(D) as shown in Figure 3. The CL counts increased in the order of (A), (B), (C), and (D). However, CL counts with (A) and (B) were very small and showed little change at the examined concentrations of Eosin Y. From the data obtained in Figure 2 and Figure 3, it was confirmed that the ternary mixed solutions of (C) and (D), especially solution (D), indicated a large response for Eosin Y based on peroxyoxalate CL. The tendency of the ternary mixed solutions, especially organic solvent-rich solution, to show large CL based on the peroxyoxalate

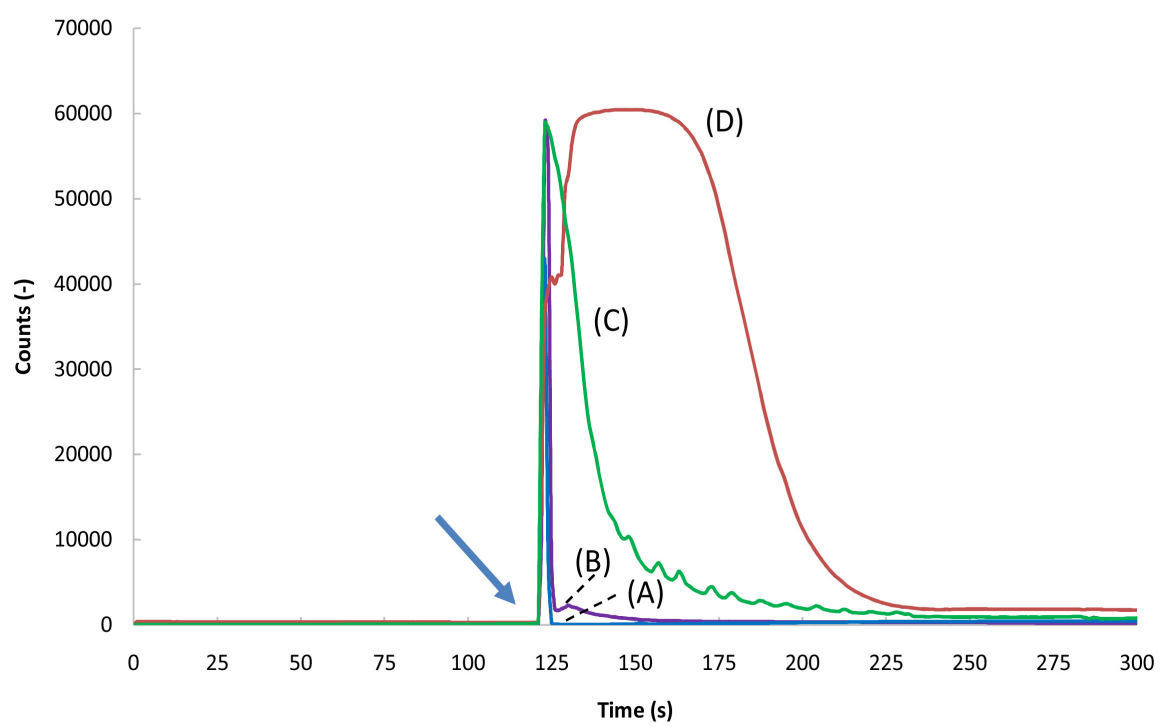

Figure 2. CL profiles of $1 \mathrm{mM}$ Eosin Y using the four solutions. Conditions: CL reagent solution, $2.0 \mathrm{mM}$ TCPO-200 $\mathrm{mM} \mathrm{H}_{2} \mathrm{O}_{2}$ mixture $(0.05 \mathrm{~mL}$, acetonitrile was used as a solvent); solutions (A)-(D) described in the figure caption of Figure 1; $2.5 \mathrm{~mL}$; and temperature, $22^{\circ} \mathrm{C}$. The arrow shows the injection point of CL reagent solution. 


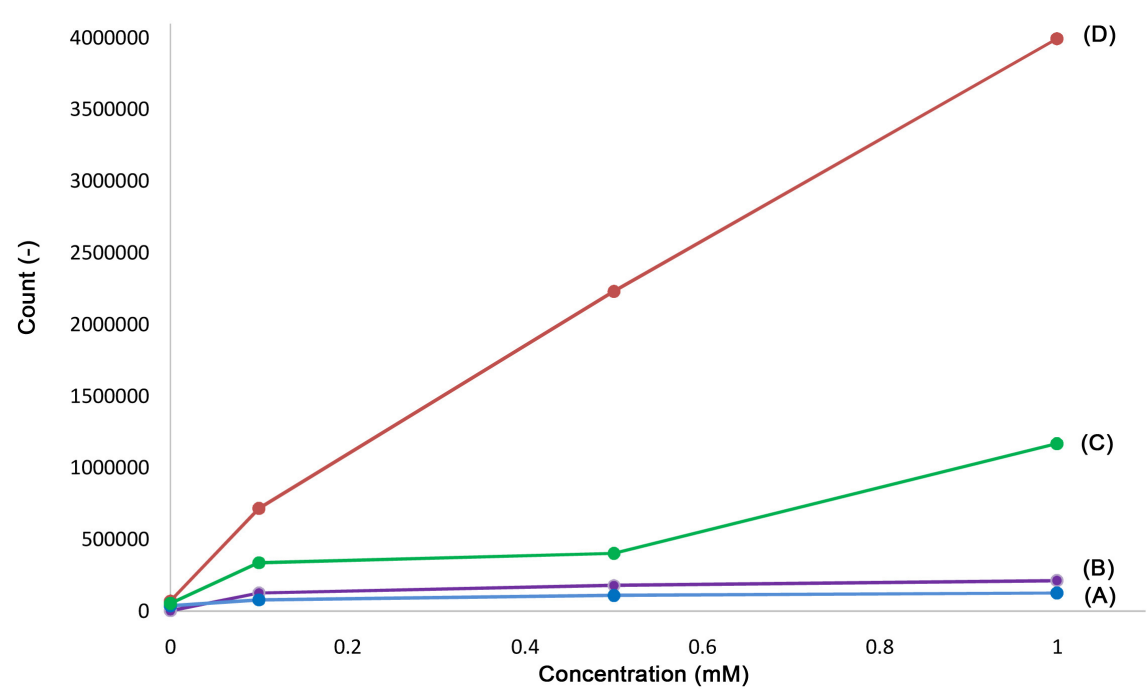

Figure 3. The CL counts of $1 \mathrm{mM}$ Eosin $\mathrm{Y}$ using the four solutions. Conditions: CL reagent solution, $2.0 \mathrm{mM}$ TCPO-200 $\mathrm{mM} \mathrm{H}_{2} \mathrm{O}_{2}$ mixture $(0.05 \mathrm{~mL}$, acetonitrile was used as a solvent); solutions (A)-(D) described in the figure caption of Figure 1; $2.5 \mathrm{~mL}$; and temperature, $22^{\circ} \mathrm{C}$.

reaction system was similar to that reported in our previous paper [12], where we examined the influence of the ternary mixed solution on a luminal CL reaction system. The effect of the ternary mixed solution on peroxyoxalate CL is discussed in the next section.

\subsection{CL of Eosin Y with Various Compositions of the Ternary Mixed Solution}

We examined the CL profiles by using various compositions of the ternary mixed solution, the water rich and organic solvent-rich, as shown in the phase diagram of Figure 4. The compositions of the water-rich mixed solutions in Figure 4 were (C) (the water-acetonitrile-ethyl acetate volume ratio, 15:3:2), (1) (15:3.2:1.8 volume ratio), (2) (15:3.4:1.6 volume ratio), and (3) (15:3.6:1.4 volume ratio). The composition of the organic solvent-rich solutions in Figure 4 were (D) (water-acetonitrile-ethyl acetate volume ratio, 3:8:4), (1)' (3.8:11.8:5.2 volume ratio), (2)' (3.6: 11.4:5.0 volume ratio), and (3)' (3.4:11.8:4.8 volume ratio). The CL profiles of $1.0 \mathrm{mM}$ Eosin $\mathrm{Y}$ by using the water-rich mixed solutions of (C) and (1)-(3) as well as the organic solvent-rich mixed solutions of (D) and (1)'-(3)' are shown in Figure 5(a) and Figure 5(b), respectively. The CL with the water-rich solutions decreased in this order: (C), (1), (2), and (3), and the CL with the organic solvent-rich solutions decreased in the order: (D), (1)', (2)', and (3)', as shown in Figure 5. The CL gradually decreased as the compositions of the ternary mixed solution moved away from the boundary curve.

There was little information for CL in the ternary water-hydrophilic/hydrophobic organic solvent mixed solution, and the reason why the ternary mixed solution enhanced CL is not yet clear. The ternary homogeneous solution near the boundary curve may cause formation of small clusters (micro-clusters) in the 


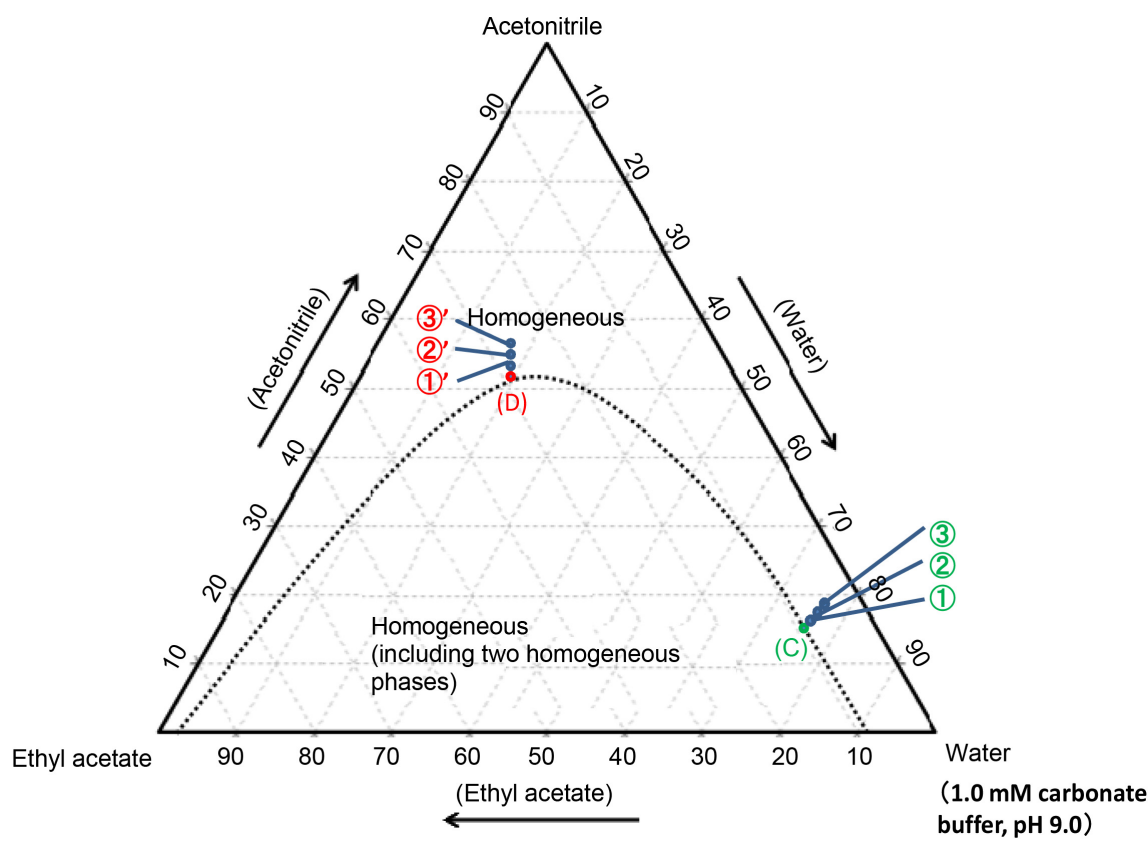

Figure 4. Phase diagram of the water-acetonitrile-ethyl acetate ternary mixed solution at $22^{\circ} \mathrm{C}$. The water-rich solutions: (C) the water-acetonitrile-ethyl acetate mixed solution (15:3:2 volume ratio; water-rich), (1) the water-acetonitrile-ethyl acetate mixed solution (15:3.2:1.8 volume ratio), (2) the water-acetonitrile-ethyl acetate mixed solution (15:3.4:1.6 volume ratio), and (3)the water-acetonitrile-ethyl acetate mixed solution (15:3.6:1.4 volume ratio). The organic solvent-rich solutions are: (D) and the water-acetonitrile-ethyl acetate mixed solution (3:8:4 volume ratio; organic solvent-rich), (1)' the water-acetonitrileethyl acetate mixed solution (3.8:11.8:5.2 volume ratio), (2)' the water-acetonitrile-ethyl acetate mixed solution (3.6:11.4:5.0 volume ratio), and (3)' the water-acetonitrile-ethyl acetate mixed solution (3.4:11.8:4.8 volume ratio).

solvent solution. The interface of the clusters, with features of hydrophilic and hydrophobic interfaces, may be effective as a CL reaction domain. The organic solvent-rich solution of (D) provided a larger CL than the water-rich solution of (C). Although the reason is not yet clear, the organic solvent-rich solution may inhibit the hydrolysis reaction of TCPO. Similar CL behavior based on the luminol reaction was reported and discussed in our previous paper [12].

\subsection{Profiles of Eosin Y and $\alpha$-Amino Acids with the Organic Solvent-Rich Ternary Solution}

The CL profiles of Eosin Y and phenylalanine ( $1 \mathrm{mM}$ each) were examined by using the organic solvent-rich ternary solution of (D). The obtained data are shown in Figure 6. Although the CL profile of phenylalanine was examined with a detection window about 5 times larger than that of Eosin Y, we could observe a sufficient CL signal even for phenylalanine as an $\alpha$-amino acid by using the specific mixed solution.

The CL counts of twenty types of $\alpha$-amino acids were examined by using the organic solvent-rich solution of (D), together with solution (A) as a reference. As alanine was not dissolved with solution (A) and (D), we could not examine it. 


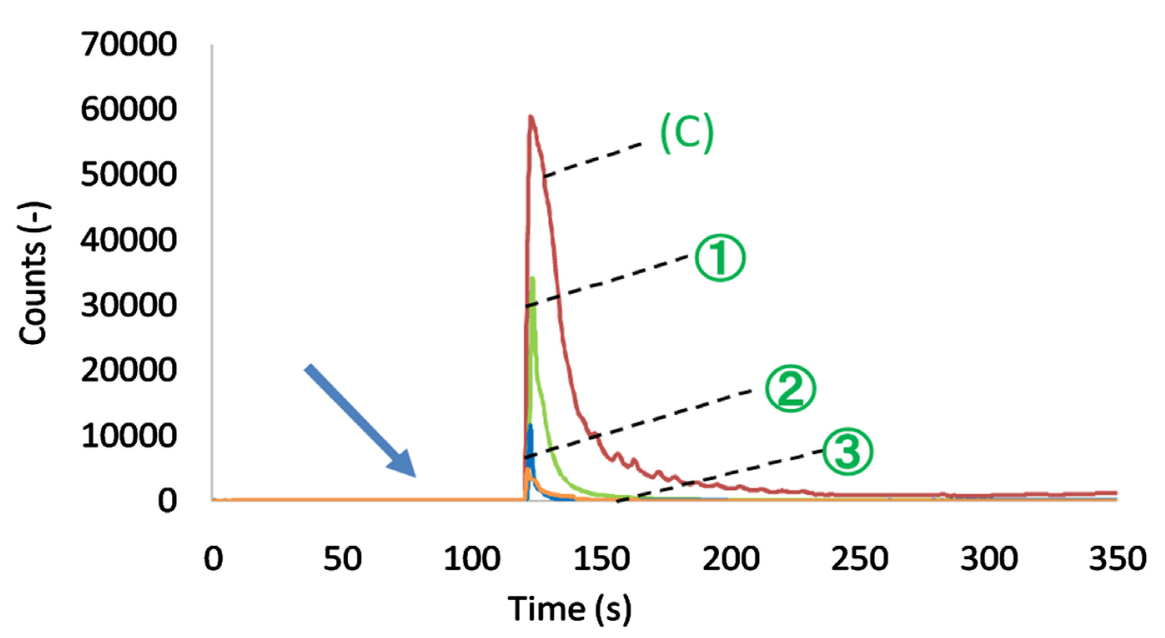

(a)

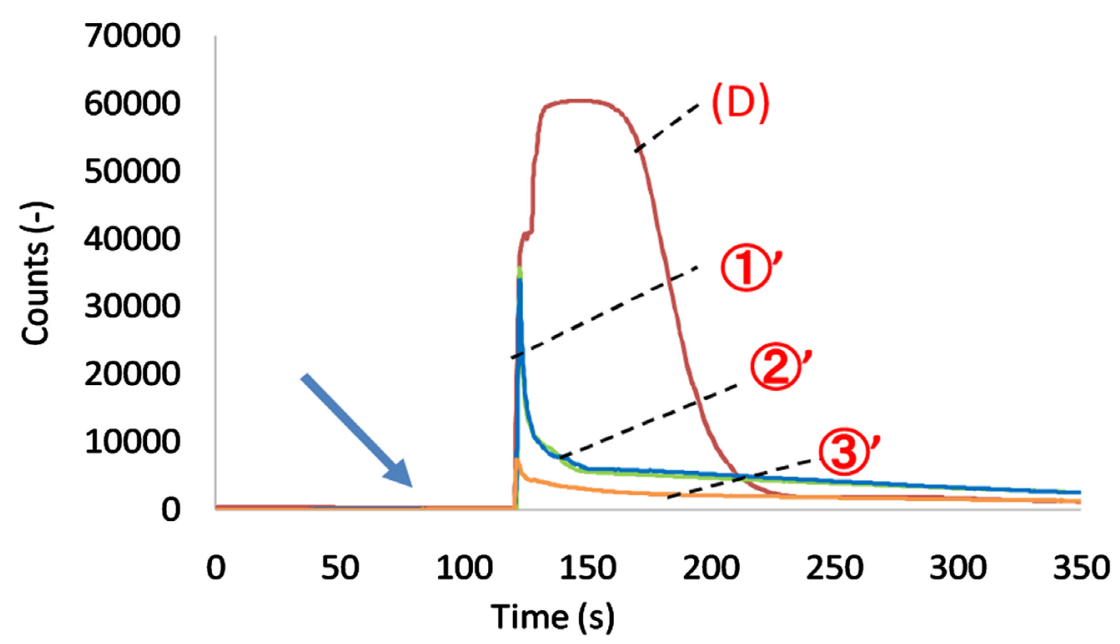

(b)

Figure 5. CL profiles of $1 \mathrm{mM}$ Eosin $\mathrm{Y}$ using the various compositions of (a) the water-rich and (b) organic solvent-rich ternary mixed solutions. The component ratios of (C), (1)-(3), (D), and (1)'-(3)' are described in the figure caption of Figure 4. The arrow shows the injection point of CL reagent solution.

The obtained data are shown in Figure 7, where the CL counts are the average values of 3 - 5 measurements. The relative standard deviations $(n=5)$ of phenylalanine, tryptophan, and tyrosine for their CL counts were $1.32 \%, 1.20 \%$, and $1.91 \%$, respectively. As can be seen in the figure, solution (D) provided larger CL counts for all $\alpha$-amino acids than solution (A), in particular for phenylalanine, tryptophan, and tyrosine, the fluorescent $\alpha$-amino acids. An enhanced effect for peroxyoxalate CL could be observed in the presence of fluorescence $\alpha$-amino acids, similar to co-existing Eosin Y. $\alpha$-Amino acids other than fluorescent $\alpha$-amino acids also showed little CL, compared to the blank values that signified CL counts obtained in the absence of $\alpha$-amino acids. As mentioned above, solution (A) was water only and solution (D) was the water-acetonitrile-ethyl acetate ternary solution (3:8:4, volume ratio; organic solvent-rich); however, solution (A) and (D) could not necessarily have proton concentrations by the effects of 


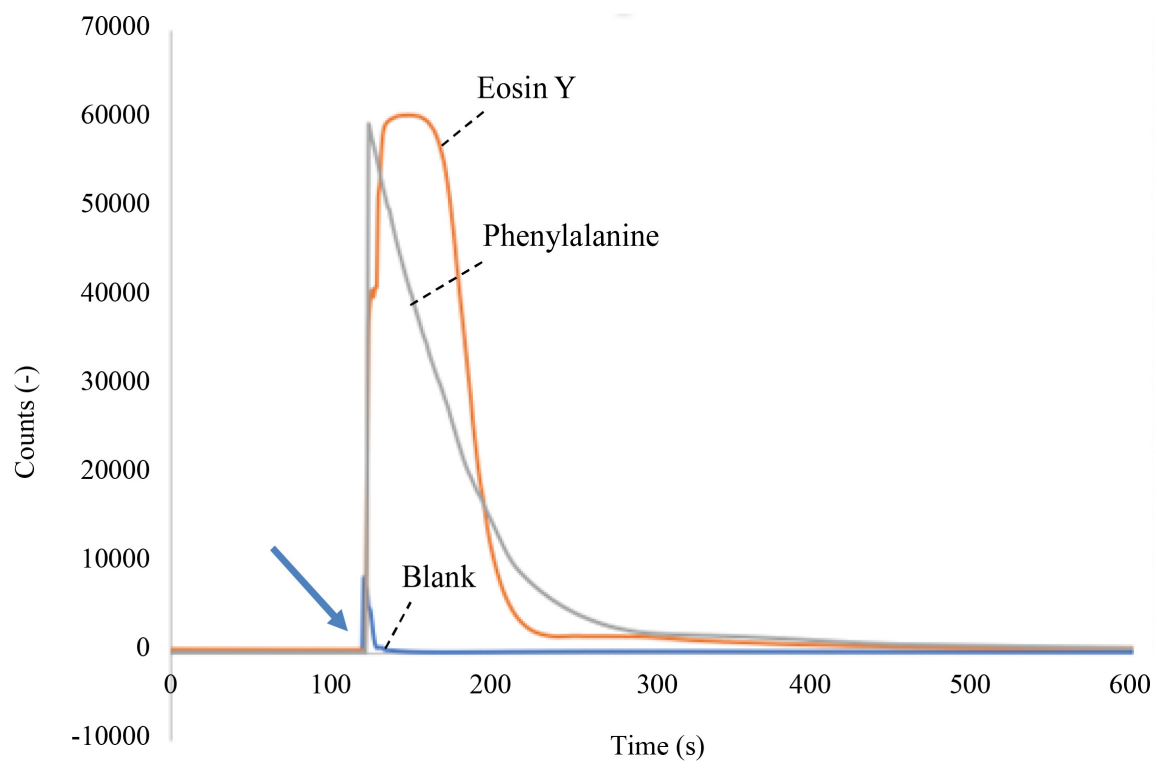

Figure 6. CL profiles of Eosin $\mathrm{Y}$ and phenylalanine using the solution (D). Conditions: CL reagent solution, $2.0 \mathrm{mM}$ TCPO-200 $\mathrm{mM} \mathrm{H}_{2} \mathrm{O}_{2}$ mixture $(0.05 \mathrm{~mL}$, acetonitrile was used as solvent); solution (D) $2.5 \mathrm{~mL}$; and temperature $22^{\circ} \mathrm{C}$. The component ratios of (D) are described in the figure caption of Figure 1. The arrow shows the injection point of CL reagent solution.

70000

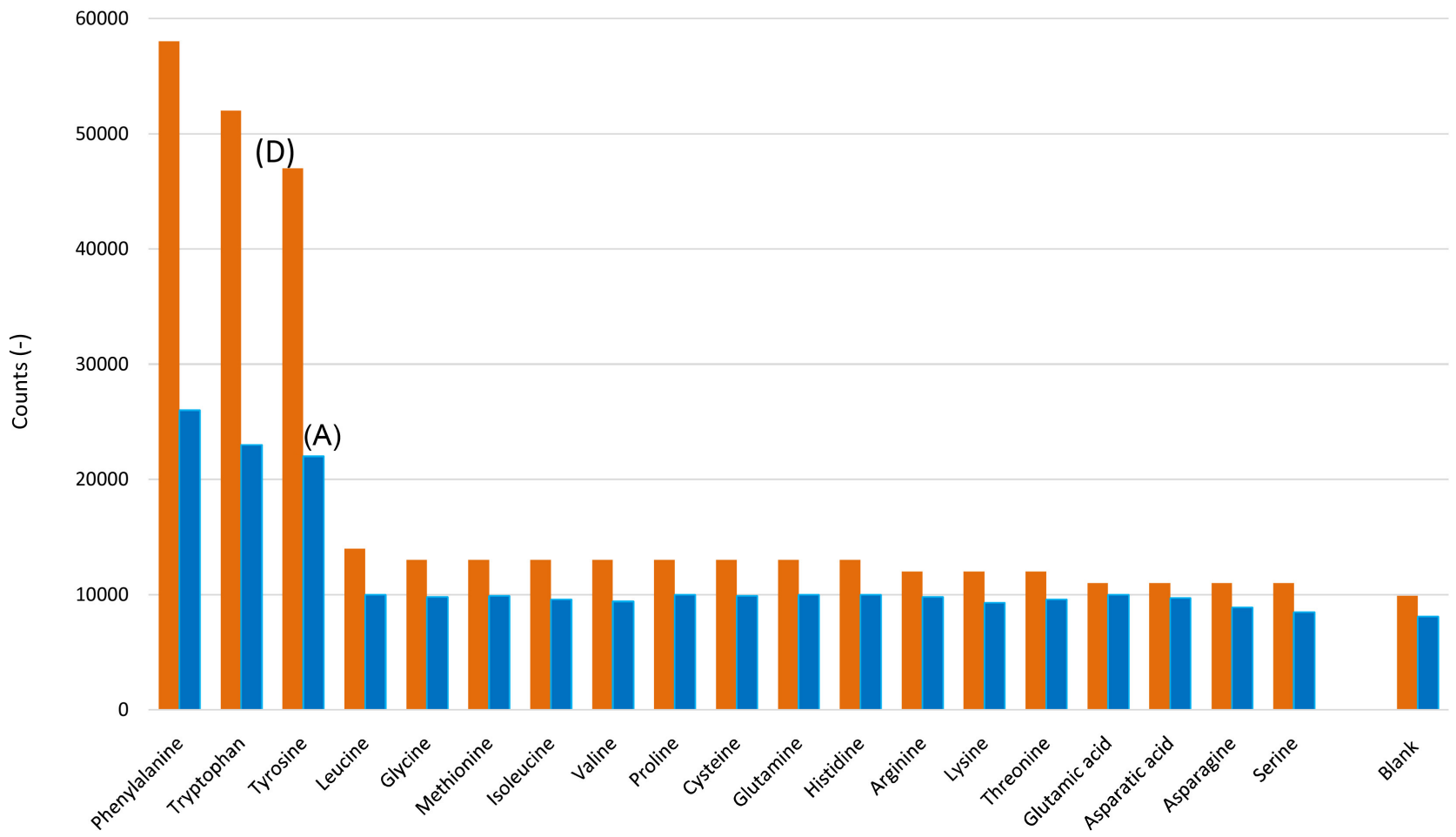

Figure 7. CL counts of $1 \mathrm{mM}$ of twenty $\alpha$-amino acids using the two solutions (A), right columns and (D), left columns. Conditions: CL reagent solution, $2.0 \mathrm{mM}$ TCPO-200 $\mathrm{mM} \mathrm{H}_{2} \mathrm{O}_{2}$ mixture $(0.05 \mathrm{~mL}$, acetonitrile was used as solvent); solutions (A) and (D) $2.5 \mathrm{~mL}$; and temperature $22^{\circ} \mathrm{C}$. The component ratios of (A) and (D) are described in the figure caption of Figure 1 . 
co-existing organic solvents. The difference in the proton concentration or the exact $\mathrm{pH}$ value between solution (A) and (D) may cause a little difference in the CL counts for sixteen-amino acids except for the three fluorescent ones between them.

\section{Conclusion}

We tried to develop a new peroxyoxalate CL system by using the ternary mixed solution of the water-acetonitrile-ethyl acetate. The CL of Eosin Y and three $\alpha$-amino acids (phenylalanine, tryptophan, and tyrosine) were enhanced with the ternary organic solvent-rich solution, compared to the CL observed with the water only and water-acetonitrile mixed solutions. The CL counts of $\alpha$-amino acids by using the ternary mixed solution of the water-acetonitrile-ethyl acetate (volume ratio 3:8:4) is almost two times than by using the buffer only solution. So it will be easier to do the detection in FIA, TRDC or other analytical systems by using the ternary mixed solution of the water-acetonitrile-ethyl acetate (volume ratio 3:8:4). The information obtained here may have important implications for future applications.

\section{Acknowledgements}

This work was supported by a Grant-in-Aid for Scientific Research (B) from the Ministry of Education, Culture, Sports, Science, and Technology, Japan (MEXT) (No. 17H03083).

\section{Conflicts of Interest}

The authors declare no conflicts of interest regarding the publication of this paper.

\section{References}

[1] Pham, A., Sedlak, D. and Doyle, F. (2012) Dissolution of Mesoporous Silica Supports in Aqueous Solutions: Implications for Mesoporous Silica-Based Water Treatment Processes. Applied Catalysis B: Environmental, 126, 258-264. https://doi.org/10.1016/j.apcatb.2012.07.018

[2] Hefter, G. (2005) Ion Solvation in Aqueous-Organic Mixtures. Pure and Applied Chemistry, 77, 605-617. https://doi.org/10.1351/pac200577030605

[3] Ruiz, D.M. and De Castro, R.E. (2007) Effect of Organic Solvents on the Activity and Stability of an Extracellular Protease Secreted by the Haloalkaliphilic Archaeon Natrialba Magadii. Journal of Industrial Microbiology \& Biotechnology, 34, 111-115. https://doi.org/10.1007/s10295-006-0174-4

[4] Tjahjono, M., Huiheng, C., Widjaja, E., Sa-Ei, K. and Garland, M. (2009) Combined On-Line Transmission FTIR Measurements and BTEM Analysis for the Kinetic Study of a Consecutive Reaction in Aqueous-Organic Phase Medium. Talanta, 79, 856-862. https://doi.org/10.1016/j.talanta.2009.05.014

[5] Murakami, N., Mizohata, M., Hashimoto, M. and Tsukagoshi, K. (2011) Fluorescence Observation Supporting Capillary Chromatography Based on Tube Radial Distribution of Carrier Solvents under Flow Conditions. Analyst, 135, 927-932. 
https://doi.org/10.1039/C0AN00820F

[6] Tsukagoshi, K. (2015) Investigation of Specific Microfluidic Flow with Two-Phase Separation Mixed Solvent Solutions and Application to Flow Technology. Journal of Flow Injection Analysis, 32, 89-95.

[7] Tsukagoshi, K. (2014) Fundamental Research and Application of the Specific Fluidic Behavior of Mixed Solvents in a Microspace. Analytical Science, 30, 65-73. https://doi.org/10.2116/analsci.30.65

[8] Yamada, K., Kan, H. and Tsukagoshi, K. (2018) Tube Radial Distribution Chromatography System Developed by Combining Commercially Available HPLC System and Open-Tubular Capillary Tube as Separation Column. Talanta, 183, 89-93. https://doi.org/10.1016/j.talanta.2018.02.046

[9] Imanishi, N., Yamashita, T., Tsukagoshi, K. and Murata, M. (2018) Phase Separation Multi-Phase Flow Using an Aqueous Two-Phase System of a Polyethylene Glycol/ Dextran Mixed Solution. Analytical Sciences, 34, 953-958. https://doi.org/10.2116/analsci.18P105

[10] Yamawaki, B., Mori, R., Tsukagoshi, K., Tsuchiya, K., Yamashita, K. and Murata, M. (2019) Microfluidic Inverted Flow of Ternary Water/Hydrophilic/Hydrophobic Organic Solvent Solution in a Y-Type Microchannel and a Proposal of the Response Microfluidic Analysis through the Experiment. Analytical Sciences, 35, 249-256. https://doi.org/10.2116/analsci.18P393

[11] Ito, Y., Tsukagoshi, K. and Kobayashi, A. (2017) Denaturation of DNA in Ternary Mixed Solution of Water/Hydrophilic/Hydrophobic Organic Solvent. Journal of Analytical Sciences, Methods and Instrumentation, 7, 40-46. https://doi.org/10.4236/jasmi.2017.72004

[12] Manai, J., Nishiyama, K. and Tsukagoshi, K. (2017) Microfluidic Analytical System with On-Line Luminol Chemiluminescence Detection Based on Annular Flow of Phase Separation Multiphase Flow. Journal of Analytical Sciences, Methods and Instrumentation, 7, 29-39. https://doi.org/10.4236/jasmi.2017.72003

[13] You, X., Li, Y., Li, B. and Ma, J. (2016) Gold Nanoclusters-Based Chemiluminescence Resonance Energy Transfer Method for Sensitive and Label-Free Detection of Trypsin. Talanta, 147, 63-68. https://doi.org/10.1016/j.talanta.2015.09.033

[14] Zou, J. and Wang, X. (1997) Bis(2,4,6-trichlorophenyl) Oxalate Chemiluminescence Reaction and Its Applications in Analytical Chemistry. Journal of Nanjing Institute of Chemical Technology, 19, 107-116.

[15] He, L., Cox, K.A. and Danielson, N.D. (1990) Chemiluminescence Detection of Amino Acids, Peptides, and Proteins Using Tris(2,2'-bipyridine)ruthenium(III). Analytical Letters, 23, 195-210. https://doi.org/10.1080/00032719008052438

[16] Yamaguchi, M., Yoshida, H. and Nohta, H. (2002) Luminol-Type Chemiluminescence Derivatization Reagents for Liquid Chromatography and Capillary Electrophoresis. Journal of Chromatography A, 950, 1-19. https://doi.org/10.1016/S0021-9673(02)00004-3

[17] Tsukagoshi, K., Nakahama, K. and Nakajima, R. (2004) Direct Detection of Biomolecules in a Capillary Electrophoresis-Chemiluminescence Detection System. Analytical Chemistry, 76, 4410-4415. https://doi.org/10.1021/ac030344i

[18] Tsukagoshi, K., Otsuka, M., Hashimoto, M., Nakajima, R. and Kondo, K. (1999) Batch-Type Detection Cell Using a Peroxyoxalate Chemiluminescence System for Capillary Electrophoresis. Analytical Sciences, 15, 1257-1260.

https://doi.org/10.2116/analsci.15.1257 